\title{
Isothermal Titration Calorimetry enables rapid characterisation of enzyme kinetics and inhibition for the human soluble Epoxide Hydrolase
}

Giancarlo Abis ${ }^{\dagger,}$, Raúl Pacheco-Goméz ${ }^{\S}$, Tam T.T. Bui ${ }^{\dagger,}$, Maria R Conte $, \uparrow, * *$

$\dagger$ Randall Centre for Cell and Molecular Biophysics, School of Basic and Medical Biosciences, King's College London, London, SE1 1UL, UK.

§Malvern Panalytical Ltd, Enigma Business Park, Grovewood Road, Malvern, WR14 1XZ, UK.

`Centre for Biomolecular Spectroscopy, King's College London, London, SE1 1UL, UK. *corresponding author: $\underline{\text { sasi.conte@,kcl.ac.uk }}$

*Present address: Division of Bioscience, Institute of Structural and Molecular Biology, University College London, London, WC1E 6BT, UK 


\section{CONTENT}

\section{Supporting methods}

Figure S1. Metabolic pathway of EpFA substrates hydrolysed by hsEH.

Figure S2. Two-injection control ITC experiments to assess product-mediated inhibition of hsEH-mediated catalysis.

Figure S3. Comparison between ITC titration profiles of EETs into hsEH CTD and into buffer alone.

Figure S4. Comparison between ITC titration profiles of EpFAs into hsEH CTD and into buffer alone.

Figure S5. Comparison of ITC experiments injecting 14(15)EET/AUDA into hsEH CTD and into buffer alone.

Figure S6. Spectrofluorometric analysis of AUDA-mediated inhibition of hsEH CTD activity.

Figure S7. Analysis of substrate concentration on kinetic rates.

Table S1. Substrate concentration used for ITC experiments

References 


\section{Supporting methods}

\section{Recombinant hsEH C-Terminal Domain (CTD) preparation}

Recombinant hsEH CTD was expressed in E. coli Ros2(DE3) cells and purified as previously described. ${ }^{3}$ After the last purification step, the enzyme was dialysed overnight at $4{ }^{\circ} \mathrm{C}$ in the reaction buffer, consisting of $50 \mathrm{mM}$ HEPES $\mathrm{pH} 7.4,300 \mathrm{mM}$ $\mathrm{NaCl}, 5 \%$ glycerol, $10 \mu \mathrm{M}$ tris(2-carboxyethyl)phosphine (TCEP). The protein was then concentrated to $1 \mathrm{mg} \mathrm{mL}-1$ and stored at $-80^{\circ} \mathrm{C}$ in small aliquots upon flash freezing in liquid nitrogen. Protein concentration was assessed using a theoretical extinction coefficient, obtained from ProtPARAM ExPASY. ${ }^{4}$

\section{Substrate and inhibitor sample preparation}

EpFAs were purchased from Bertin Pharma, dissolved in ethanol. The ethanol was evaporated using a Savant SpeedVac (ThermoScientific) at $30^{\circ} \mathrm{C}$ for 3 hours. Substrate stock solutions were prepared dissolving the lipids to a final concentration of $1 \mathrm{mg} \mathrm{mL}^{-1}$ in the identical reaction buffer used for protein dialysis, to avoid heat effects due to differences in buffer composition and/or $\mathrm{pH}$ (buffer mismatch effects). Solutions were stored in small aliquots at $-20^{\circ} \mathrm{C}$ for a maximum time of four weeks.

The inhibitor AUDA (12-[[(tricyclo[3.3.1.13,7]dec-1-ylamino)carbonyl]amino]dodecanoic acid - Sigma-Aldrich) was dissolved in dimethyl sulfoxide (DMSO Sigma) and diluted to the desired final concentration in the reaction buffer (final DMSO concentration of $1.3 \%$ ).

\section{Substrate single-injection ITC kinetics theoretical background}

The theoretical basis of kinetic rates determination by ITC has been previously described. ${ }^{5-8}$ In brief, in the ITC experiment, the reaction rate (aka heat rate $\mathrm{d} Q / \mathrm{d} t$ ) is described by the heat variation $(\mathrm{d} Q)$ over time $(\mathrm{d} t)$. The total heat variation measured by the calorimeter is the sum of all the events occurring during the reaction under analysis, which include the catalysis, the interaction between substrate and enzyme (and putative cofactors), and the proton release or uptake from the buffer. The total heat measured in an ITC experiment is proportional to the enthalpy of all these molecular events, the apparent enthalpy $\left(\Delta \mathrm{H}_{\mathrm{app}}\right)$, and to the number of moles of product generated $(n)$, which in turn is given by the total volume multiplied by the concentration of product (eq 1):

$$
Q=n \Delta \mathrm{H}_{\mathrm{app}}=\mathrm{V}[\mathrm{P}] \Delta \mathrm{H}_{\mathrm{app}}
$$


The reaction rate $v(\mathrm{~d}[\mathrm{P}] / \mathrm{d} t)$, can be related to the amount of heat generated over the same time $(\mathrm{dQ} / \mathrm{d} t)$ through eq 2 :

$$
v=\frac{\mathrm{d}[\mathrm{P}]}{\mathrm{d} t}=\frac{1}{\mathrm{~V} \Delta \mathrm{H}_{\mathrm{app}}} \cdot \frac{\mathrm{d} Q}{\mathrm{~d} t}
$$

In single-injection ITC measurements, saturating concentrations of substrate (greater than the Michaelis-Menten constant $\mathrm{K}_{\mathrm{M}}$ ) are injected in an enzyme solution. The injection generates negative or positive heat signals, and the heat rate is monitored in a continuous manner, until the signal returns to baseline (reference power), indicating that the substrate is completely depleted, and the enzymatic reaction is complete. The $\Delta \mathrm{H}_{\mathrm{app}}$ is measured by the integration of the single peak:

$$
\Delta \mathrm{H}_{\mathrm{app}}=\frac{1}{[\mathrm{~S}]_{0} \mathrm{~V}} \int_{t=0}^{t=\infty} \frac{\mathrm{d} Q}{\mathrm{~d} t} \mathrm{~d} t
$$

where $[\mathrm{S}]_{0}$ is the total substrate concentration in the single injection experiments. $[\mathrm{S}]_{i}$ can be therefore extrapolated at any given time $i$ from the integral of heat evolved (eq 3).

By determining the $v_{i}$ from eq 2 and the $[\mathrm{S}]_{i}$ from eq 3 at any given time, plots of reaction rate versus substrate concentration, corresponding to Michaelis-Menten plots, can be obtained:

$$
v=\frac{d[\mathrm{P}]}{d \mathrm{t}}=\frac{\mathrm{k}_{\mathrm{cat}}[\mathrm{E}]_{\mathrm{tot}}[\mathrm{S}]}{\mathrm{K}_{\mathrm{M}}+[\mathrm{S}]}
$$

where $[E]_{\text {tot }}$ is the total concentration of enzyme. Eq 4 provides affinity for the substrate $\left(\mathrm{K}_{\mathrm{M}}\right)$, turnover rate $\left(\mathrm{k}_{\mathrm{cat}}\right)$, and catalytic efficiency $\left(\mathrm{k}_{\mathrm{cat}} / \mathrm{K}_{\mathrm{M}}=\mathrm{K}_{\mathrm{sp}}\right)$ values.

\section{Isothermal titration calorimetry (ITC) set-up}

ITC experiments were performed on MicroCal PEAQ-ITC and MicroCal iTC200 calorimeters (Malvern), both set up to high-feedback mode, reference power $5 \mu \mathrm{cal}$ $\mathrm{sec}^{-1}$, stirring speed of $750 \mathrm{rpm}$, and experimental temperature $25^{\circ} \mathrm{C}$. A 600 seconds pre-injection delay was applied for baseline stabilisation after equilibration.

\section{Substrate single-injection ITC kinetics experiments}

Preliminary test experiments were carried out to determine the final measurement parameters, including temperature, substrate-to-enzyme ratio concentration, injection volume, reference power and injection speed. Particular attention was paid to 
substrate-to-enzyme ratio concentration, by measuring the enzymatic rate reaction at increasing concentrations of each substrate, until no changes in $\mathrm{k}_{\mathrm{cat}}\left(\right.$ and $\mathrm{K}_{\mathrm{sp}}$ ) were observed, indicating that steady state was reached (Fig. S7). The optimised experiments were performed as follows: hsEH CTD and substrate were diluted in identical reaction buffer to final concentrations of $250 \mathrm{nM}$ and $0.5-1.5 \mathrm{mM}$ respectively (Table S1). The enzyme solution was transferred to the sample cell, and the substrate solution was loaded in the injection syringe. After the calorimeter had equilibrated and the stabilising pre-injection delay, one single $38 \mu \mathrm{L}$ injection was performed with a speed in the $0.58-0.76 \mu \mathrm{L} \mathrm{sec}-1$ range, followed by a spacing of 1000-4000 seconds (according to the reaction rate measured). Each substrate was tested in at least three biological repeats. Substrate autohydrolysis and heat of dilution phenomena were assessed by replacing the enzyme solution in the calorimetric cell with reaction buffer and carrying out the measurements with identical experimental setting. This control experiment also evaluates other important effects, such as plunger friction and mixing of the components in the calorimetric cell. Product inhibition for each hsEH CTD-mediated hydrolytic reaction was evaluated by comparing the apparent reaction enthalpy and peak heat rate (measured as described in ${ }^{12}$ ) of two successive $19 \mu \mathrm{L}$ injections in 25 seconds, spaced by 1000-1500 seconds. Apparent enthalpy of the reaction, heat rate $(\mathrm{d} Q / \mathrm{d} t)$ and Michaelis-Menten plots were generated using the 'Enzyme kinetics - single injection' model within the MicroCal PEAQ-ITC Analysis Software (Malvern), following the guidelines in the software user manual.

\section{Progressive inhibition ITC enzyme kinetics measurements}

To evaluate the inhibitory constant $\left(\mathrm{K}_{\mathrm{i}}\right)$ of hsEH antagonists and their mode of inhibition we employed a previously developed progressive inhibition $\operatorname{method}^{9}$ and optimised it for our system, as follows. As a test inhibitor, we used the well-known hsEH antagonist AUDA. A solution containing $0.5 \mathrm{mM}$ of 14(15)EET and $67.37 \mathrm{nM}$ of AUDA was prepared in reaction buffer. The solution was then loaded into the instrument syringe. hsEH CTD was diluted in reaction buffer to a final concentration of $250 \mathrm{nM} ; 1.3 \%$ (v/v) DMSO was then added to the hsEH CTD solution to match the substrate/inhibitor titrant mixture. hsEH was then transferred to the calorimeter sample cell. After baseline stabilisation, four $9.5 \mu \mathrm{L}$ injections of 12.5 seconds each were performed, at a speed of $0.76 \mu \mathrm{L} \mathrm{sec}-1$, followed by 1000 seconds spacing. 
Substrate autohydrolysis and heat of dilution phenomena were assessed by injecting the 14(15)EET/AUDA solution into reaction buffer, using the same experimental parameters. Apparent reaction enthalpy, $\mathrm{d} Q / \mathrm{d} t$ rates and Michaelis-Menten parameters of each injection peak were generated using the 'Enzyme kinetics - single injection' model within the MicroCal PEAQ-ITC Analysis Software (Malvern), as described above. Note that in this case the $K_{M}$ values obtained were apparent $\left(K_{M}{ }^{\prime}\right)$, because of the presence of the inhibitor. ${ }^{2} \mathrm{~K}_{\mathrm{M}}$ ' was plotted versus inhibitor concentration, following the methodology established by Di Trani et al. ${ }^{9,10}$ The data were fitted to a 'straight line' equation in GraphPad and were further considered exclusively if $\mathrm{R}^{2}$ was $\geq 0.9$. For a competitive inhibitor, the $y$ axis intercept gives the true $K_{M}$, whilst the slope gives $K_{M}^{1} / K_{i}$ ratio, where $K_{i}$ is the inhibition constant. The experiment was repeated three times.

\section{Inhibitory constant measurements with spectrofluorometric method}

AUDA inhibitory potency was also tested with a spectrofluorometric method which monitors the hsEH-mediated hydrolysis of the synthetic substrate PHOME (3-phenylcyano(6-methoxy-2-naphthalenyl)methyl ester-2-oxiraneacetic acid), through the detection of the fluorescent end-product $6 \mathrm{M} 2 \mathrm{~N}$ (6-methoxy-2-naphthaldehyde). ${ }^{11}$ Recombinant hsEH CTD protein was diluted in a 96-well polystyrene microtiter plate (Thermo Scientific), in freshly prepared $25 \mathrm{mM}$ Tris- $\mathrm{HCl} \mathrm{pH} 7.4$ to a final concentration of $15 \mathrm{nM}$. The enzyme was incubated at room temperature for 10 minutes with the inhibitor (diluted $1: 40 \mathrm{v} / \mathrm{v}$ to obtain increasing concentration between 1 and $250 \mathrm{nM}$ AUDA). A fresh $0.4 \mathrm{mM}$ solution of PHOME was prepared in DMSO and diluted 1:40 v/v in the 96-well plate. The hsEH-mediated hydrolysis was monitored with a POLARstar Omega spectrofluorometer (BMG Labtech) for 20 minutes every 45 seconds, at $30^{\circ} \mathrm{C}$ temperature and 750 gain. The excitation wavelength was fixed at $330 \mathrm{~nm}$, the emission detection at $460 \mathrm{~nm}$. Measurements were performed in four replicates and each experiment was repeated six times. The relative fluorescence units (RFUs) readouts were corrected for the PHOME autohydrolysis background signal, and converted into nmol of $6 \mathrm{M} 2 \mathrm{~N}$ using a conversion curve built as previously reported. ${ }^{3}$ The nmol $_{6 \mathrm{M} 2 \mathrm{~N}}$ detected at 12 minutes were normalised against the product measured in the absence of inhibitor, and plotted, as percentage of activity, against the $\log _{10}$ of AUDA concentration. Half maximal inhibitory concentration values (IC50) were obtained by fitting of the data point with 
' $\log$ (inhibitor) vs. response - variable slope' in GraphPad, and further considered exclusively if $\mathrm{R}^{2}$ was $\geq 0.9$. Inhibitory constant $\left(\mathrm{K}_{\mathrm{i}}\right)$ values were calculated using the Cheng-Prusoff equation, ${ }^{2}$ using the Michaelis-Menten constant $\left(\mathrm{K}_{\mathrm{M}}\right)$ for PHOME previously reported. ${ }^{1}$ 


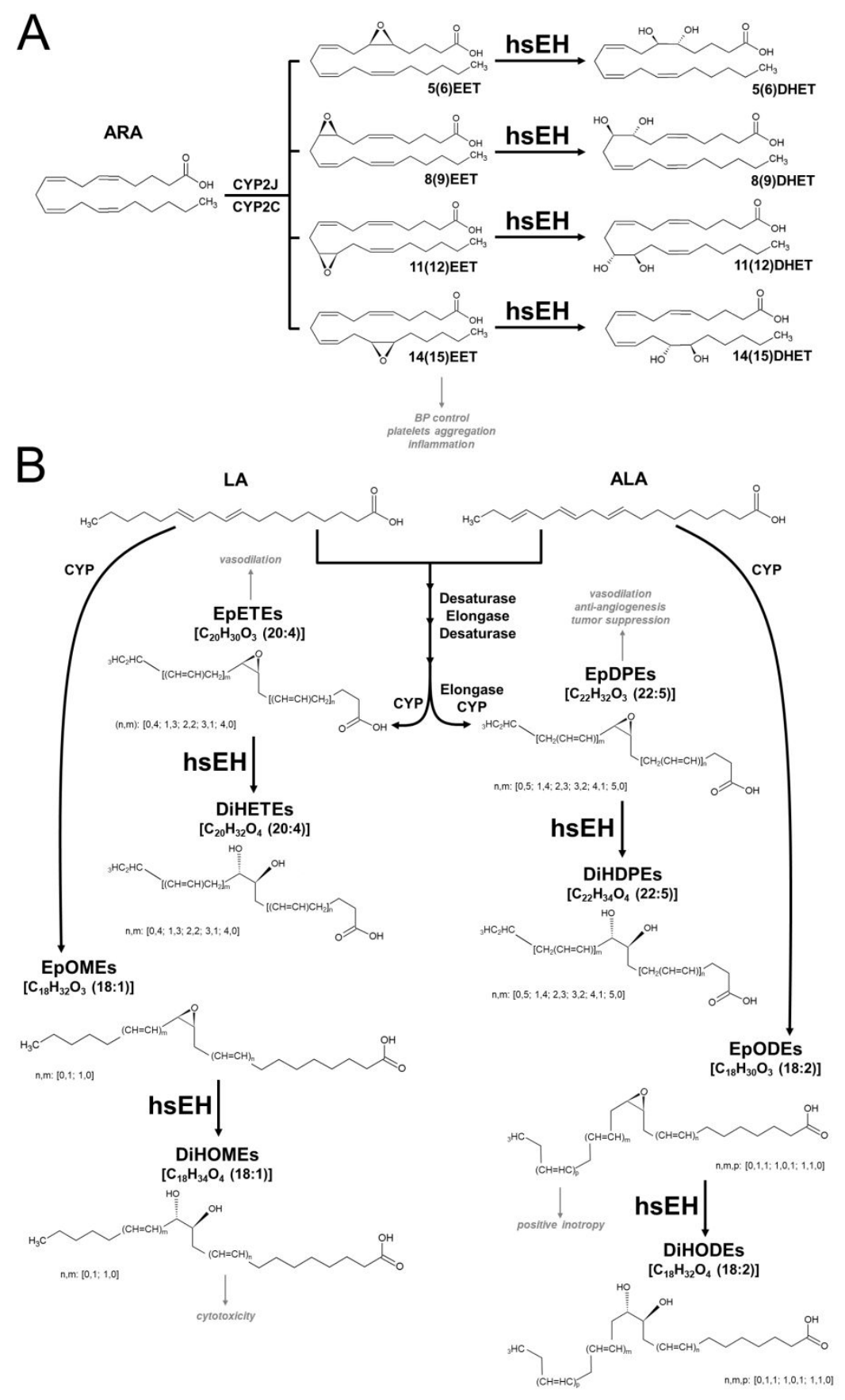

Figure S1. EpFA substrates hydrolysed by hsEH. (A) Metabolic pathway of EETs. EETs are generated via direct epoxidation of arachidonic acid (ARA, 20:4 n-6) by two cytocrome P-450 oxygenases, (CYP) 2C and 2J. (B) EpFAs derived by linoleic acid (LA, 18:2 n-6) and $\alpha$-linolenic acid (ALA, 18:3 n-3). EpOMEs and EpODEs are produced either via direct epoxidation by CYP enzymes or by non-enzymatic reaction with reactive oxygen species of linoleic and $\alpha$-linoleic acids. Both fatty acids compete for endogenous desaturation, elongation, and CYP-dependent oxygenation to EpETEs and EpDPEs. Abbreviations: ARA: arachidonic acid; EETs: epoxyeicosatrienoic acids; DHETs: dihydroxy-eicosatrienoic acids; LA: linoleic acid; ALA: $\alpha$-linoleic acid; EpOMEs: epoxyoctadecenoic acids; DiHOME: dihydroxy-octadecenoic acid; EpETE: epoxyeicosatetraenoic acids; DiHETEs: dihydroxy-eicosatetraenoic acids; EpDPE: epoxydocosapentaenoic acids; DiHDPEs: dihydroxy-docosapentaenoic acids; EpODEs: epoxyoctadecadienoic acids; DiHODEs: dihydroxy-octadecadienoic acids. 


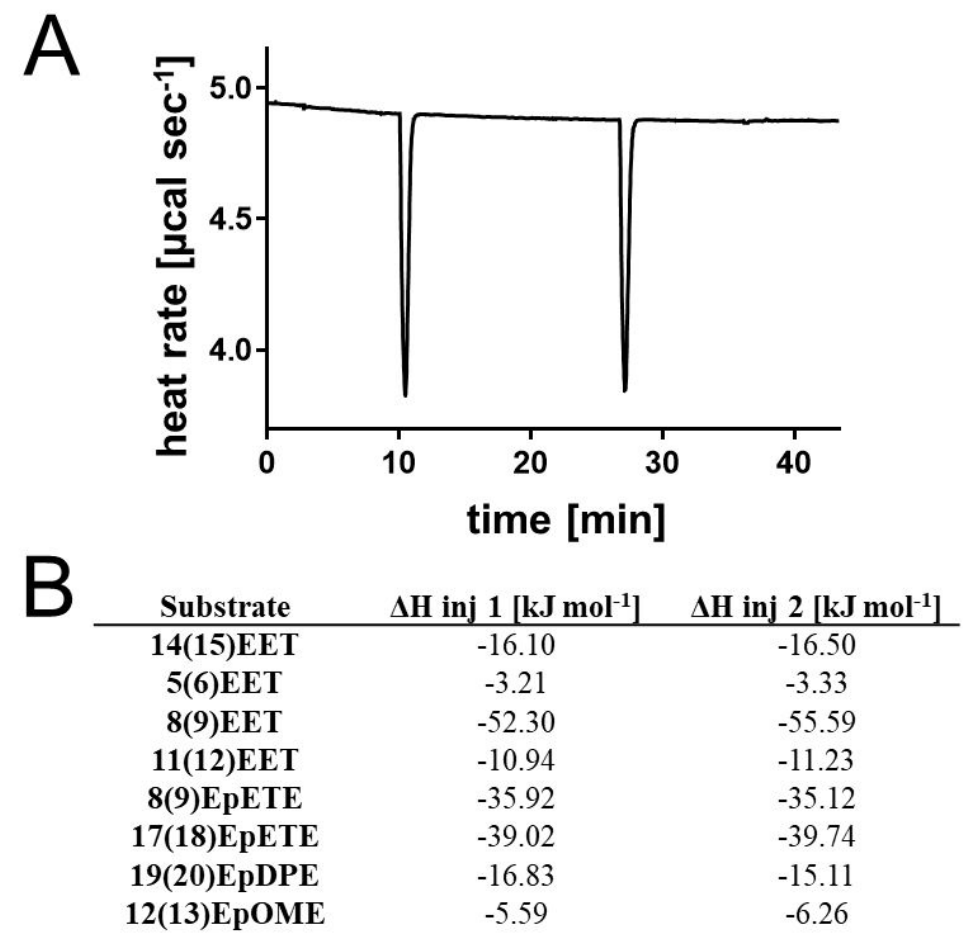

\begin{tabular}{|c|c|c|}
\hline Substrate & $\begin{array}{l}\text { Peak Heat Rate } \\
\text { inj } 1\left[\mu \mathrm{cal} \mathrm{sec} \mathrm{sec}^{-1}\right]\end{array}$ & $\begin{array}{l}\text { Peak Heat Rate } \\
\text { inj } 2\left[\mu \mathrm{cal} \mathrm{sec} \mathrm{sec}^{-1}\right]\end{array}$ \\
\hline 14(15)EET & 3.8291 & 3.8471 \\
\hline 5(6)EET & 4.8801 & 4.8924 \\
\hline 8(9)EET & 4.2582 & 4.2376 \\
\hline 11(12)EET & 4.5400 & 4.5580 \\
\hline 8(9)ЕрЕTЕ & 4.3840 & 4.4246 \\
\hline 17(18)ЕрЕTE & 4.7731 & 4.8093 \\
\hline 19(20)EpDPE & 4.6093 & 4.6381 \\
\hline 12(13)ЕрОМЕ & 4.6687 & 4.6376 \\
\hline
\end{tabular}

Figure S2. Two-injection ITC experiment to assess any product-mediated inhibition of hsEH catalysis. (A) Thermogram of the two-injections of 14(15)EET into hsEH CTD. The virtually identical profile of the two injection peaks indicates no 14(15)DHET-mediated inhibition of the epoxy-hydrolysis. (B) Enthalpy and (C) peak heat rate quantification of the two-injection ITC experiment. For all the EpFAs used in this study a two-injection experiment was performed as control (not shown). Enthalpy and peak heat rate values measured from injection (inj) 1 and 2 were virtually identical in all cases, indicating that the products of the hydrolysis do not exert inhibitory effects on hsEH catalysis. 
A

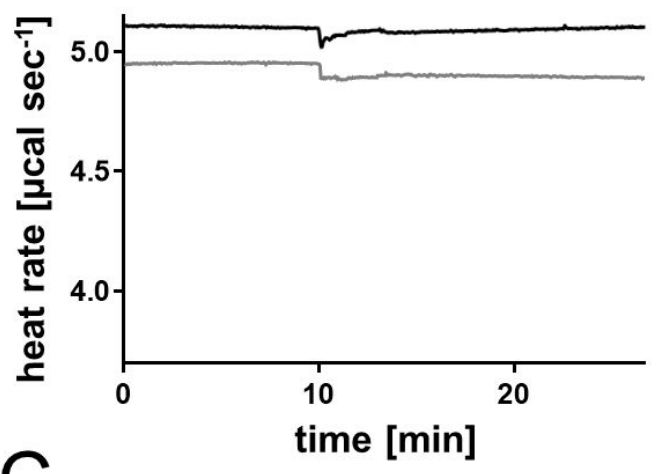

C

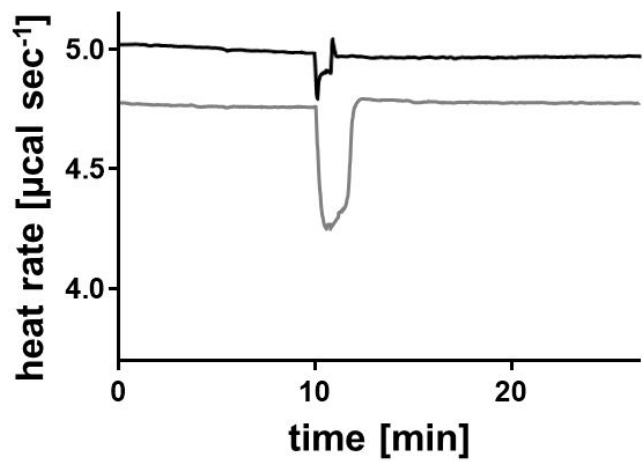

B

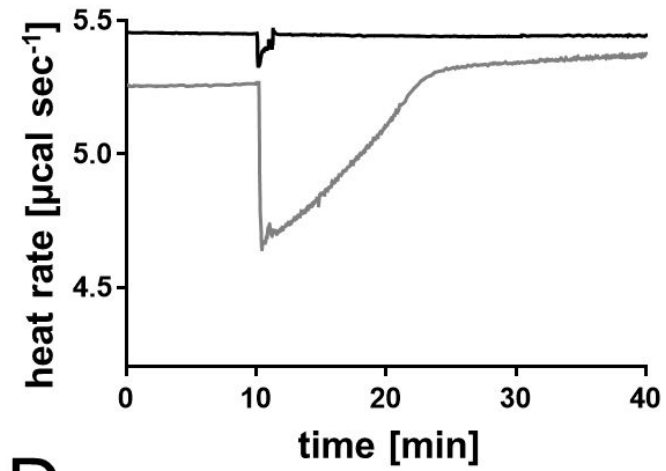

$\mathrm{D}$

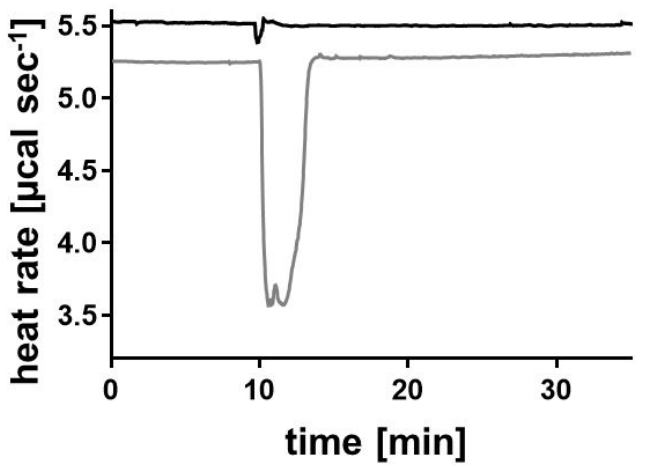

Figure S3. Comparison between ITC titration profiles of EETs into hsEH CTD (grey) and into buffer alone (black). (A) 5(6)EET; (B) 8(9)EET; (C) 11(12)EET; (D) 14(15)EET. The injection of all the regioisomeric EETs into buffer alone gave rise to negligible heat of dilution and did not show substrate autohydrolysis, indicating that the thermal profiles observed in the presence of hsEH CTD are generated from enzymatic catalysis. 

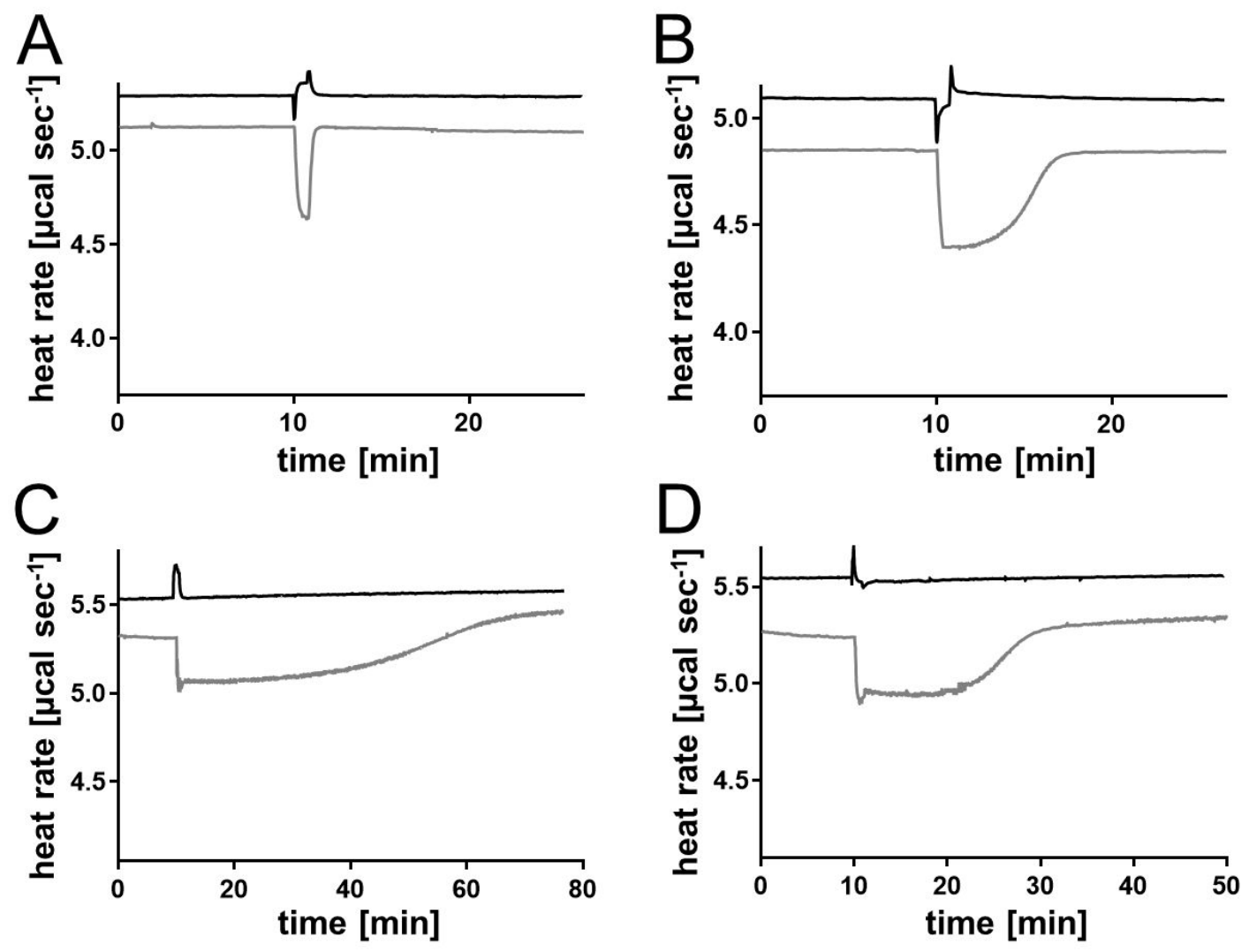

Figure S4. Comparison between ITC titration profiles of EpFAs into hsEH CTD (grey) and into buffer alone (black). (A) 11(12)EpOME; (B) 8(9)EpETE; (C) 17(18)EpETE; (D) 19(20)EpDPE. Injection into buffer alone of all the EpFAs herein analysed generated negligible heat of dilution and did not produce autohydrolysis, indicating that the thermal profiles observed in the presence of hsEH CTD are generated by enzymatic catalysis. 


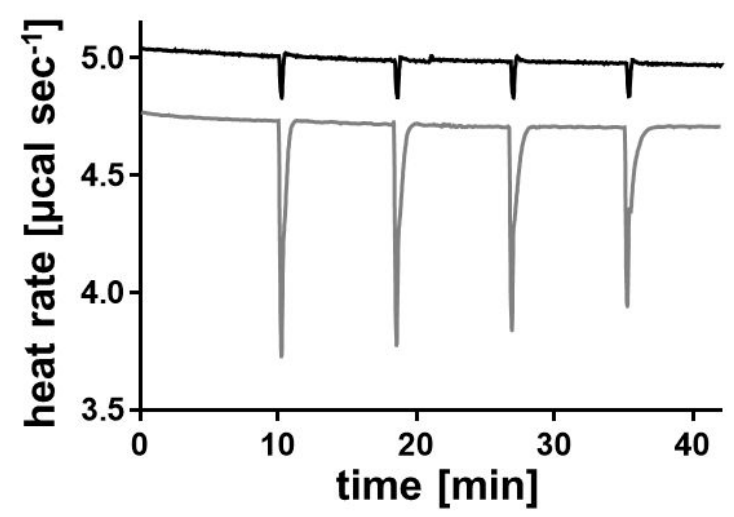

Figure S5. Comparison of ITC experiments injecting 14(15)EET/AUDA into hsEH CTD (grey) and into buffer alone (black). The injection of the mixture of substrate and inhibitor into buffer did not generate autohydrolysis phenomena, indicating that the thermal profiles observed in the presence of hsEH CTD are the result of enzyme activity. 

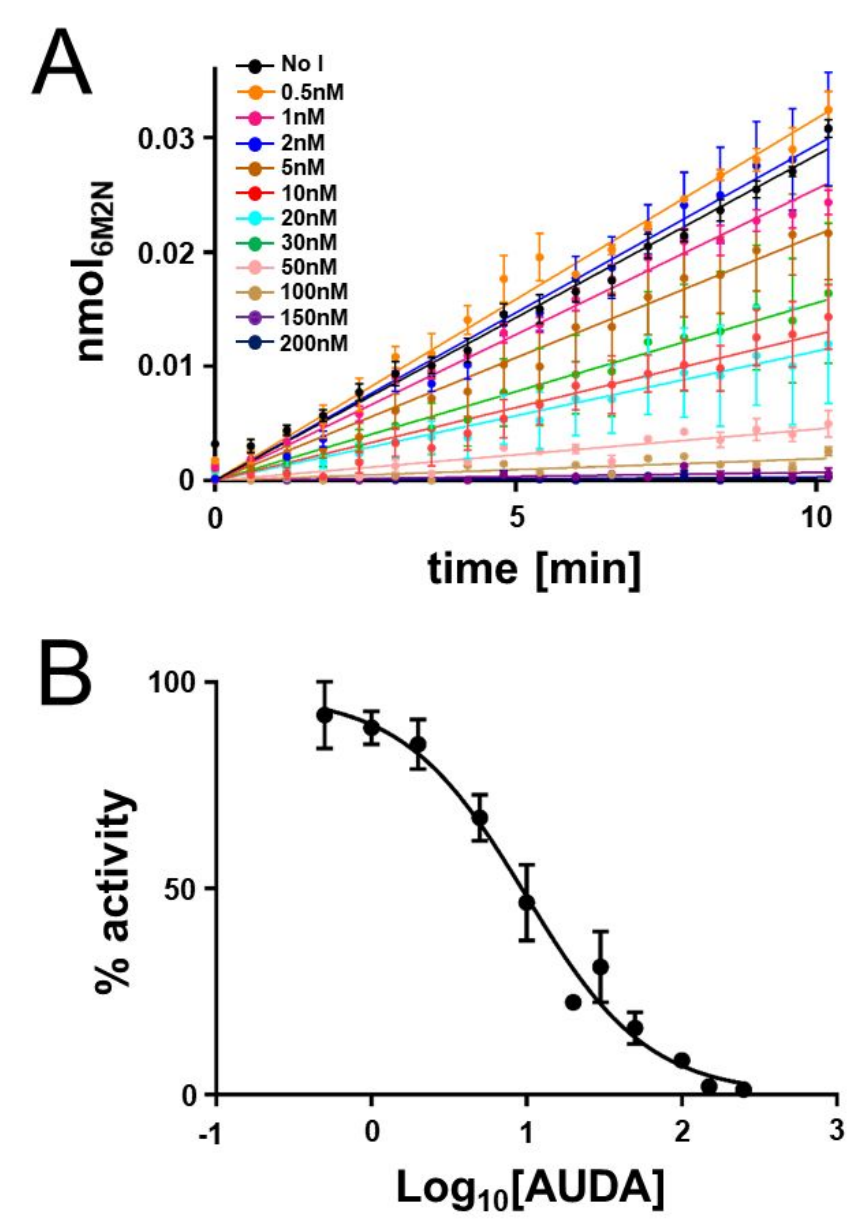

Figure S6. Spectrofluorimetric analysis of AUDA-mediated inhibition of hsEH CTD activity. (A) Representative 6M2N spectrofluorimetric detection. Increased AUDA concentration in solution resulted in lower end-product detection. (B) IC50 plot derived from data in (A). The curve was built with the percentage of activity calculated with increasing AUDA concentrations, as previously reported ${ }^{1}$. The IC50 $(14.23 \pm 2.21 \mathrm{nM})$ is the concentration at which the curve intercepts the $50 \%$ inhibition level. Using the Cheng-Prusoff equation ${ }^{2}$ and the $\mathrm{K}_{\mathrm{M}}$ for PHOME that we previously reported in identical experimental conditions $(3.99 \pm 0.34 \mu \mathrm{M})^{1}$, an average $\mathrm{K}_{\mathrm{i}}$ of $4.99 \pm 0.71 \mathrm{nM}$ for AUDA was obtained. 

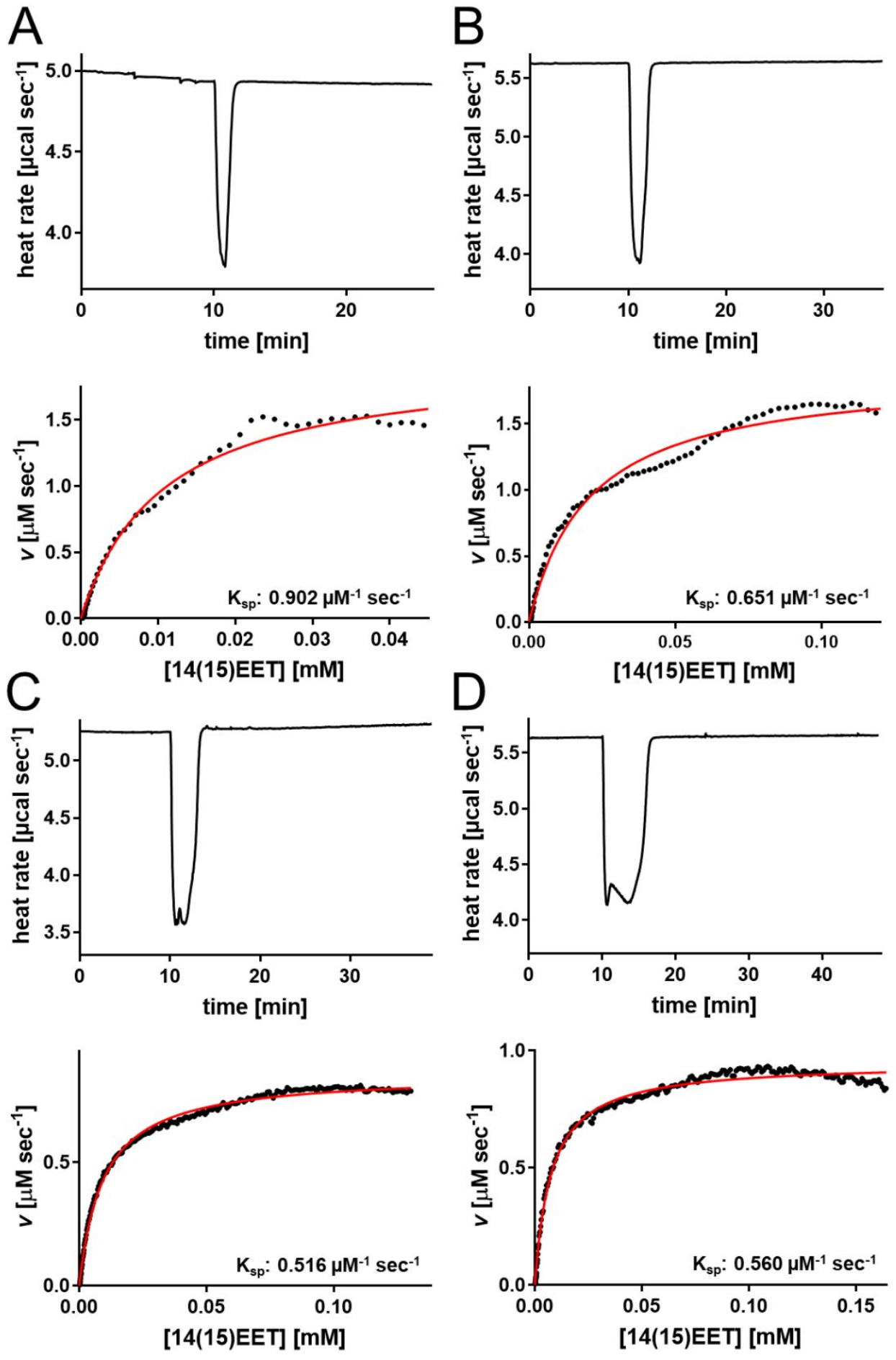

Figure S7. Analysis of substrate concentration on kinetic rates. Increasing the concentration of 14(15)EET used in the experiments yielded enzyme saturation, enabling the analysis of the reaction rate in steady-state. The four panels correspond to: $0.5 \mathrm{mM}$ (A), $1 \mathrm{mM}$ (B), $1.5 \mathrm{mM}$ (C) and $3 \mathrm{mM}$ (D) of 14(15)EET injected into $250 \mathrm{nM}$ hsEH CTD. The $\mathrm{K}_{\mathrm{sp}}$ values indicated no difference between the experiments using 1.5 and $3 \mathrm{mM}$ 14(15)EET, suggesting that the former condition (equivalent to a final concentration of substrate-to-enzyme in the cell of approximately 8 times the $K_{M}$ value) yielded enzyme saturation. Similar experiments were performed per each substrate analysed in this study. 


\begin{tabular}{cc} 
Substrate & Concentration \\
\hline 5(6)EET & $0.5 \mathrm{mM}$ \\
8(9)EET & $0.75 \mathrm{mM}$ \\
11(12)EET & $0.5 \mathrm{mM}$ \\
14(15)EET & $1.5 \mathrm{mM}$ \\
8(9)EpETE & $0.5 \mathrm{mM}$ \\
17(18)EpETE & $1.5 \mathrm{mM}$ \\
19(20)EpDPE & $1.5 \mathrm{mM}$ \\
12(13)EpOME & $0.5 \mathrm{mM}$
\end{tabular}

Table S1. Substrate concentration used in the ITC syringe for the experiments presented in Figs 1, 2, 3 and Supplementary Figs S3 and S4. 


\section{References}

(1) Abis, G.; Charles, R. L.; Kopec, J.; W Yue, W.; Atkinson, R. A.; Bui, T. T.; Lynham, S.; Popova, S.; Sun, Y.-B.; Fraternali, F.; Eaton, P.; Conte M. R. 15deoxy- $\Delta^{12,14}$-Prostaglandin $\mathrm{J}_{2}$-mediated inhibition reveals two allosteric sites of the human soluble Epoxide Hydrolase. Commun. Biol. https://doi.org/10.1038/s42003-019-0426-2.

(2) Copeland, R. A. Evaluation of enzyme inhibitors in drug discovery, First.; Wiley, 2005.

(3) Abis, G.; Charles, R. L.; Eaton, P.; Conte, M. R. Expression, purification, and characterisation of human soluble Epoxide Hydrolase (hsEH) and of its functional C-terminal domain. Protein Expr. Purif. 2019, 153 (July 2018), 105-113. https://doi.org/10.1016/j.pep.2018.09.001.

(4) Gasteiger, E.; Hoogland, C.; Gattiker, A.; Duvaud, S.; Wilkins, M. R.; Appel, R. D.; Bairoch, A. Protein Identification and Analysis Tools on the ExPASy Server. Proteomics Protoc. Handb. 2005, 571-607. https://doi.org/10.1385/1592598900.

(5) Mazzei, L.; Ciurli, S.; Zambelli, B. Isothermal Titration Calorimetry to Characterize Enzymatic Reactions, 1; Elsevier Inc., 2016, 567. 215-36. https://doi.org/10.1016/bs.mie.2015.07.022.

(6) Luo, Q.; Chen, D.; Boom, R. M.; Janssen, A. E. M. Revisiting the enzymatic kinetics of pepsin using isothermal titration calorimetry. Food Chem. 2018, 268 (June), 94-100. https://doi.org/10.1016/j.foodchem.2018.06.042.

(7) Hansen, L. D.; Transtrum, M. K.; Quinn, C.; Demarse, N. Enzyme-catalyzed and binding reaction kinetics determined by titration calorimetry. Biochim. Biophys. Acta - Gen. Subj. 2016, 1860 (5), 957-966. https://doi.org/10.1016/j.bbagen.2015.12.018.

(8) Todd, M. J.; Gomez, J. Enzyme kinetics determined using calorimetry: a general assay for enzyme activity? Anal. Biochem. 2001, 296 (2), 179-187. https://doi.org/10.1006/abio.2001.5218.

(9) Di Trani, J. M.; Moitessier, N.; Mittermaier, A. K. Complete Kinetic Characterization of Enzyme Inhibition in a Single Isothermal Titration Calorimetric Experiment. Anal. Chem. 2018, 90 (14), 8430-8435. https://doi.org/10.1021/acs.analchem.8b00993.

(10) Di Trani, J. M.; Moitessier, N.; Mittermaier, A. K. Measuring Rapid TimeScale Reaction Kinetics Using Isothermal Titration Calorimetry. Anal. Chem. 2017, 89 (13), 7022-7030. https://doi.org/10.1021/acs.analchem.7b00693.

(11) Wolf, N. M.; Morisseau, C.; Jones, P. D.; Hock, B.; Hammock, B. D. Development of a high-throughput screen for soluble epoxide hydrolase inhibition. Anal. Biochem. 2006, 355, 71-80. https://doi.org/10.1016/j.ab.2006.04.045.

(12) Chan, W. K.; Mason, M.; Hansen, L. D.; Kenealey, J. D. Measuring Enzymatic Stability by Isothermal Titration Calorimetry. J. Vis. Exp. 2019, 145, e59302, https://doi:10.3791/59302. 